\title{
Effect of hip position and breathing pattern on abdominal muscle activation during curl-up variations
}

\author{
Soo-Han Kim, Se-Yeon Park* \\ Department of Physical Therapy, College of Health Science, Kaya University, Gimhae, Korea
}

Recent studies have indicated that applying different breathing pattern and changes of extremity positions could be a useful way of performing challenging exercises that include coactivation of the abdominal muscles. The aim of this study was to investigate the effects of changes of breathing pattern and hip position on abdominal muscle activity during curl-up exercises. Surface electromyography responses of the rectus abdominis (RA), internal oblique (IO), external oblique (EO) muscles and rectus femoris (RF), and Borg rating of perceived exertion (Borg RPE) score were investigated during the curl up exercises. A total of 20 healthy, physically active male volunteers completed two hip positions $\left(45^{\circ}\right.$ and $90^{\circ}$ hip flexion) and three breath types (slow expiration, maximal inspiration, and maximal expiration) for curl-up variations. The curl-

\section{INTRODUCTION}

In the field of sport rehabilitation, achieving abdominal muscle strength and proper order of activation of the abdominal muscles are major considerations for improving performance and functional movement, as well as for preventing musculoskeletal lower back pain (Cho et al., 2014; Davidson and Hubley-Kozey, 2005; Hartvigsen et al., 2015). Various exercises are used to strengthen the abdominal muscles, including curl-ups, sit-ups, plank exercises, and exercises using suspension or unstable devices (Comfort et al., 2011; Czaprowski et al., 2014; Hartvigsen et al., 2015). Although available evidence suggests that multijoint full body exercises, such as plank with suspension, may be better for gymnastics training rather than core-specific exercises (Martuscello et al., 2013), low-intensity exercises, and those that require minimal movement of the extremities, such as curl-ups, are recommended for rehabilitation (Ishak et al., 2016; Rutkowska-Kucharska and up with $90^{\circ}$ of hip flexion significantly increased the muscular activities of $10, \mathrm{EO}, \mathrm{RF}$, and Borg RPE score $(P<0.05)$. The curl-up with maximal expiration significantly elevated the $I 0$ and EO activity, compared to other breathing conditions. There were no significant difference in RA activities $(P>0.05)$. The present study showed both the hip position and breathing pattern affect abdominal muscle activities during the curl-up exercise, especially of the oblique muscles. Among the breathing patterns investigated herein, the curl-up with maximum expiration produced greater activation of the oblique muscles compared to slow expiration and maximum inspiration.

Keywords: Abdominal strengthening, Curl-up, Electromyography, Training
*Corresponding author: Se-Yeon Park (ib https://orcid.org/0000-0002-5769-8732 Department of Physical Therapy, Kaya University, 208 Samgye-ro, Gimhae 50830, Korea

Tel: +82-55-344-5295, Fax: +82-55-344-5285, E-mail: arclain@kaya.ac.kr Received: March 26, 2018 / Accepted: April 19, 2018
Szpala, 2018).

A curl-up is a common low-intensity abdominal strengthening exercise that places no stress on the lumbar spine. Previous researchers have suggested variations to the curl-up exercise to maximize its strengthening effects (Rutkowska-Kucharska and Szpala, 2010). It was previously demonstrated that extension of the upper extremity and flexion of the lower extremity confers advantages with respect to rectus abdominis (RA) activation during curl-up exercises (Rutkowska-Kucharska and Szpala, 2010). Burden and Redmond (2013) reported that curl-ups performed with the feet restrained are advantageous for activating abdominal muscles. In another study, in which muscle activities during curl-ups performed at different trunk flexion angles were examined, greater flexion angle induced more activity of the upper RA, while a reduced flexion angle was advantageous for activating the lower RA (Kim et al., 2015). More recently, Lee et al. (2016) suggested that restriction of neck flexion could generate greater activity of the 
RA and external oblique (EO) abdominis (Lee et al., 2016).

Although researchers have previously investigated the effects of variations of the curl-up on abdominal muscle activities, in general, these variations (in trunk flexion angle) have increased the external rather than internal moment. Anatomically, the abdominal muscles not only flex the trunk, but also assist in control of the breathing (Muscolino, 2012). The abdominal muscle in front of the abdominal cavity is extended or shortened following inspiration or expiration. The clinical literature suggests that abdominal muscles are the principal muscles involved in forced expiration (Ishida and Watanabe, 2015). However, few studies have examined the effects of inspiration and expiration control on abdominal muscle activities (Ishida and Watanabe, 2015; Yoon et al., 2014). The internal oblique (IO)-transversus abdominis was highly activated by slow and controlled expiration during curl-up exercises (Yoon et al., 2014). More recently, Ishida and Watanabe (2015) demonstrated that maximum expiration could be advantageous for activating the RA, EO, and IO muscles during side bridge exercises. To our knowledge, three breathing control patterns, quiet inspiration, slow expiration, and maximum expiration, have been investigated to date. However, there is lack of study which the breathing control is superior to other methods during curl-up exercises.

In contrast to the sit-up, the curl-up exercise slightly elevates the upper extremity relative to the lower extremity. Thus, the action of the hip flexor may not be considered. However, one of the hip flexor muscles, the iliopsoas muscle, is initiated from the upper lumbar body such that the hip joint angle, which determines the length of the iliopsoas, indirectly affects the activities of the abdominal muscles. Rutkowska-Kucharska and Szpala (2010) compared abdominal muscle activities according to hip position during curl-up exercises; however, it is necessary to further examine the effects of hip position on the abdominal muscles.

The curl-up exercise is commonly used by physiotherapists, and in athletic training programs, to strengthen the abdominal muscles (Comfort et al., 2011; Czaprowski et al., 2014). To the best of our knowledge, no study has evaluated abdominal muscle activities according to the three breathing patterns noted above, nor according to hip position. Therefore, the primary purpose of the present study was to compare quiet inspiration, slow expiration and maximal expiration with respect to abdominal muscle activation during curl-up exercises. The secondary purpose was to determine whether a flexed hip joint position can effectively stimulate the abdominal muscles. We hypothesized that abdominal muscle activities would differ among the three breathing patterns, and that activation would be greater with increased hip joint flexion during curl-up exercises.

\section{MATERIALS AND METHODS}

\section{Participant}

The sample size in present study was determined using G-power 3.1.3 software. Installing a significant level of 0.05 , power of 0.8 using convenience sampling, participants were recruited from a local university. As an inclusion criterion, it was determined as participant with no musculoskeletal disorder during recent 6 months. For preventing the potential influence of fatty tissue on measuring surface electromyographic activity, participants with body mass index $(\mathrm{BMI}) \geq 25 \mathrm{~kg} / \mathrm{m}^{2}$ were excluded.

A total of 20 male individuals participated in this study. Demographics of final sample participants were 20-26 years old $(22.55 \pm 1.85$, mean \pm standard deviation) with height and weight of $175.19 \pm 6.01 \mathrm{~cm}$ and $67.56 \pm 5.94 \mathrm{~kg}$, respectively. The average BMI was $22.07 \pm 1.36 \mathrm{~kg} / \mathrm{m}^{2}$. All participants gave informed written consent according to the protocol approved by Kaya University Faculty of Health Science Human Ethics Committee (approval number: 201712 204).

\section{Instrumentation}

Surface electromyography (sEMG) data were collected using a wireless EMG system (Free EMG 300, BTS Bioengineering, Garbagnate Milanese, Italy). The sEMG signals were sampled with a 1,000-Hz frequency. The data obtained were computerized with the EMG acquisition software (BTS Bioengineering). Four surface electrodes were attached parallel to the muscle fibers on the dominant right side as follows: on the RA muscle at approximately 3 $\mathrm{cm}$ lateral to the umbilicus; on the $\mathrm{EO}$ abdominis muscle at the anterior end of the eighth rib; on the IO muscle at approximately $1 \mathrm{~cm}$ medial to the anterior superior iliac spine; and on the rectus femoris (RF) muscle at approximately the midpoint at the line between anterior superior iliac spine and patella (Cram et al., 1998; Czaprowski et al., 2014). Skin preparation of shaving and cleaning with alcohol was done for according to the SEINAM (Surface ElectroMyoGraphy for the Non-Invasive Assessment of Muscles) recommendation.

\section{Procedures}

Present study entitled two hip positions and three breath types for curl up variations. For preventing muscular fatigue 3-min of rest time between trials, and 5-min of rest time and acclimation 
to the each exercise variation are given to participant.

For the $45^{\circ}$ hip flexion and $90^{\circ}$ knee flexion which is same as general curl-up, subject was lying as supine position and primal researcher measured hip and knee angle with goniometer. For the curl up with $90^{\circ}$ of hip and knee flexion, several boxes were stacked as making hip as $90^{\circ}$. Commonly, subject's knee and feet were fixed to primal researcher (Fig. 1). Each subject performed curl-up exercise with using three different breathing controls. For the slow expiration, subject elevated their upper body with slowly exhaling their breath. For the maximal inspiration pattern, subject inhale the air as maximal as they can in their initial supine position. Then, subject elevated their upper body without exhaling air. For the maximal expiration pattern, subject in their supine position exhale the air as maximal as they can. Then subject elevate their upper body without inhaling or exhaling air. During six variations of curl up exercises, height adjustable rod was installed beside the table for equalizing elevating upper body height. This rod was set above nipple, and the height was set when the inferior angle of the scapulae is parted from the bed surface during general curlup. Six curl-up variations were randomly assigned, and subjective difficulty was investigated just after each curl-up exercise.

Before collecting sEMG data during exercise variations, each subject performed two trials at maximal voluntary muscle contraction (MVIC) for the included muscles against manual resistance. The highest value between the two MVIC trials was used for the normalization procedure. The normalized values of RA, $\mathrm{EO}, \mathrm{IO}, \mathrm{RF}$ were presented as \%MVIC. The values including normalized surface EMG, Borg rating of perceived exertion (Borg RPE) scale values were used for statistical analysis.

\section{Statistical analysis}

The root mean square of sEMG data during the test was obtained with a widow length of $0.125 \mathrm{sec}$ and averaged. The data are expressed as the \%MVIC relative to normalized data. PASW Statistics ver. 18.0 (SPSS Inc., Chicago, IL, USA) was used to determine the significance of differences in \% MVIC values between
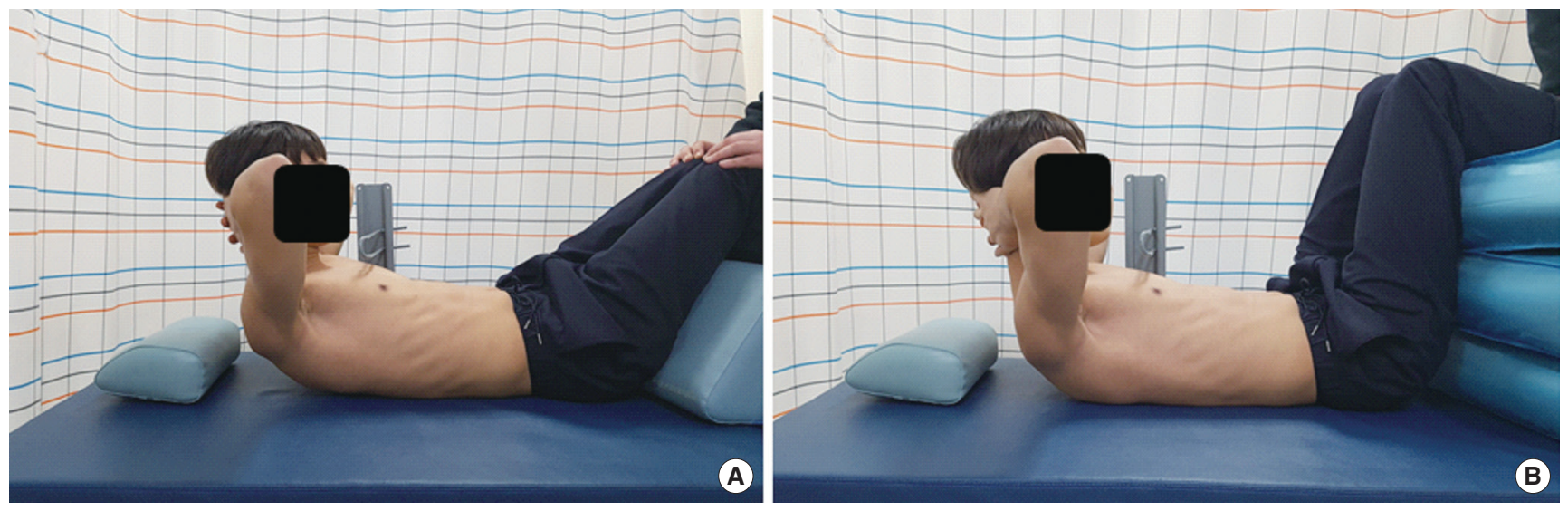

Fig. 1. Demonstration of the curl-up with $45^{\circ}$ hip flexion (A), and curl-up with $90^{\circ}$ hip flexion (B).

Table 1. Descriptive statistics of normalized electromyography data (\%MVIC) of the four muscles and Borg rating of perceived exertion (Borg RPE) score during sit-up variations

\begin{tabular}{|c|c|c|c|c|c|c|}
\hline \multirow{2}{*}{ Variable } & \multicolumn{3}{|c|}{ Hip $45^{\circ}$ flexion - curl-up } & \multicolumn{3}{|c|}{ Hip $90^{\circ}$ flexion - curl-up } \\
\hline & Slow-Exp & Max-Insp & Max-Exp & Slow-Exp & Max-Insp & Max-Exp \\
\hline RA & $49.36 \pm 14.51$ & $51.22 \pm 14.92$ & $54.59 \pm 19.29$ & $50.77 \pm 16.45$ & $51.72 \pm 16.48$ & $52.48 \pm 17.36$ \\
\hline $10^{*}$ & $36.92 \pm 18.68$ & $35.49 \pm 16.27$ & $46.98 \pm 21.91$ & $48.67 \pm 12.22$ & $47.57 \pm 16.03$ & $62.06 \pm 23.08$ \\
\hline EO* & $50.61 \pm 14.37$ & $48.82 \pm 14.46$ & $57.12 \pm 16.22$ & $65.18 \pm 24.83$ & $63.95 \pm 20.32$ & $70.74 \pm 20.57$ \\
\hline $\mathrm{RF}^{*}$ & $19.24 \pm 10.68$ & $17.73 \pm 10.00$ & $18.37 \pm 10.40$ & $28.04 \pm 16.02$ & $27.98 \pm 19.79$ & $34.98 \pm 28.66$ \\
\hline Borg RPE* & $9.45 \pm 1.93$ & $10.40 \pm 2.39$ & $12.15 \pm 2.66$ & $12.00 \pm 2.17$ & $12.75 \pm 2.51$ & $13.80 \pm 2.76$ \\
\hline
\end{tabular}

Value are presented as mean \pm standard deviation.

MVIC, maximum voluntary isometric contraction; Slow-Exp, slow expiration; Max-Insp, maximal inspiration; Max-Exp, maximal expiration; RA, rectus abdominis; I0, internal oblique abdominis; EO, external oblique abdominis; RF, rectus femoris.

${ }^{*} P<0.05$, significant difference in exercise conditions. 
test conditions. For identifying the normal distribution of data, the Kolmogorov-Smirnov test has been done before the statistical analysis. Two-way repeated-measure analysis of variance was performed to test for differences in \%MVIC for each muscle activity. For the main effects derived via pairwise multiple comparisons, the Bonferroni correction was performed to identify specific differences between exercise variations. In all analyses, $P<0.05$ was taken to indicate statistical significance.

\section{RESULTS}

The mean values of normalized EMG data and Borg RPE score were represented in Table 1 . The factor of hip position significantly affected the muscular activities of IO, EO, RF, and Borg RPE score, which were significantly higher with curl up with $90^{\circ}$ of hip flexion, compared to the curl-up with $45^{\circ}$ hip flexion $(P<$ 0.05).

The factor of breath pattern showed significant difference in IO, EO, and Borg RPE score $(P<0.05)$. Curl-up with maximal expiration significantly elevated the IO activity, compared to both curl-up with slow expiration and maximal inspiration (Fig. 2) $(P<0.05)$. The normalized sEMG data for the EO differed significantly between curl-up with maximal expiration and curl up with maximal inspiration $(F[1.17]=12.08$, observed power $=0.905)$ (Fig. 2). The curl-up with maximal expiration also showed significantly greater value of Borg RPE score than the curl-up with slow expiration (Fig. 3). There were no significant difference in
RA activities, and correlation between factors $(P>0.05)$

\section{DISCUSSION}

This study examined the effects of different breathing patterns and hip joint flexion angles on the activation of the abdominal muscles during, and subjective difficulty of, curl-up exercises. The curl-up exercise with $90^{\circ}$ hip flexion elicited more $\mathrm{IO}$ and $\mathrm{EO}$ activation and was considered more difficult. A previous biomechanical study investigated spinal flexion and pelvic rotation during curl-up exercises (Gidaris et al., 2009): curl-up with $90^{\circ}$ hip flexion could prevent iliacus and psoas activity, thus contributing to an absence of pelvic rotation during the exercise. Andersson et al. (1997) reported that, for a sit-up exercise done with the hip in a flexed position, abdominal muscle activation was greater during the initial phase. In the present study, the curl-up exercise generated limited trunk flexion, similar to previous reports.

Interestingly, RA activity did not differ significantly by hip position in this study. These results are inconsistent with those of previous studies demonstrating greater RA activity or external moment when performed on certain types of surfaces. According to a previous study, changing the internal moment (e.g., hip position) produced results similar to those of the present study. Parfrey (2008) reported no significant difference in RA activity between sit-up exercises performed with a bent knee versus those done with the leg extended. Similarly, it has been suggested that extension of the upper extremity is advantageous for stimulating the
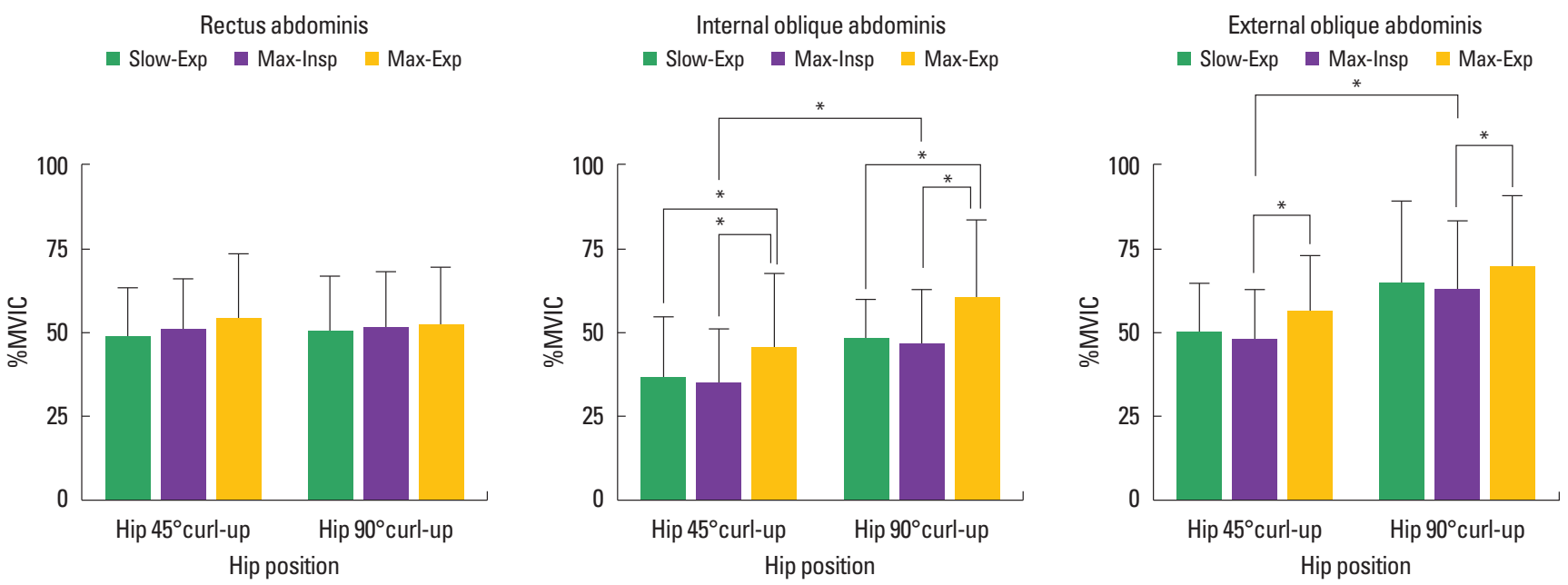

Fig. 2. The normalized electromyography data (\%MVIC) of the rectus abdominis, internal oblique abdominis, and external oblique abdominis during sit-up exercise variations. MVIC, maximum voluntary isometric contraction; Slow-Exp, slow expiration; Max-Insp, maximal inspiration; Max-Exp, maximal expiration. ${ }^{*} P<0.05$, significant difference between conditions. 

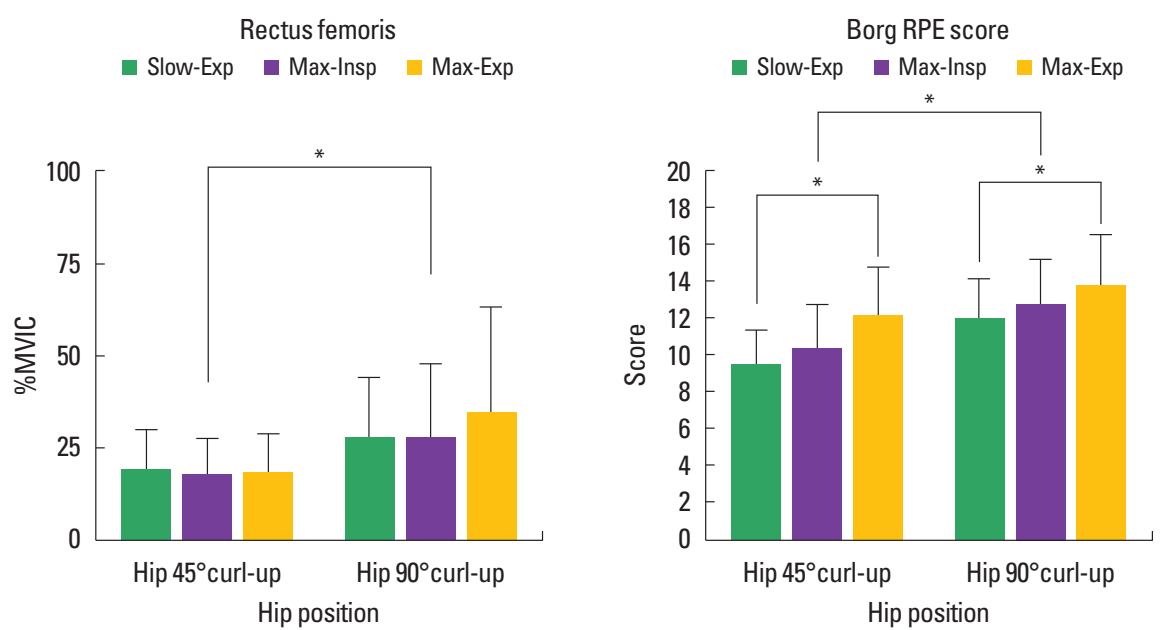

Fig. 3. The normalized electromyography data (\%MVIC) of the rectus femoris and Borg rating for perceived exertion (Borg RPE) score during sit-up exercise variations. MVIC, maximum voluntary isometric contraction; Slow-Exp, slow expiration; Max-Insp, maximal inspiration; Max-Exp, maximal expiration. ${ }^{*} P<0.05$, significant difference between conditions.

RA muscle, while variation of the lower extremity position did not significantly affect RA activity (Rutkowska-Kucharska and Szpala, 2010). The results obtained both in the present and previous studies indicate that altering the hip position does not affect RA activity.

Three breathing patterns were examined in the present study: slow expiration, maximum inspiration, and maximum expiration. These breathing patterns significantly affected $\mathrm{IO}$ and $\mathrm{EO}$ activities during curl-up exercises, as well as Borg RPE scale scores. Among the abdominal muscles, the oblique muscle fibers showed the greatest response to alterations in the breathing pattern, consistent with a previous study (Ishida and Watanabe, 2015; Yoon et al., 2014). Abe et al. (1996) suggested that, during resting ventilation, the transverse abdominis is the most active muscle, followed by the IO and EO muscles, and finally by the RA. Notably, the curl-up exercise with maximum expiration increased IO and $\mathrm{EO}$ activities in this study. Although studies investigating the effects of different breathing patterns on muscular activities during exercise are lacking, in one report the side bridge exercise done with maximum expiration increased abdominal muscle activity compared to resting expiration (Ishida and Watanabe, 2015). Furthermore, in an earlier report, adopting a supine position and performing maximum expiration thickened the abdominal muscle compared to the drawing maneuver (Ishida and Watanabe, 2015).

Another study showed that Borg RPE scale can serve as an affordable and valid tool for monitoring exercise intensity (Scherr et al., 2013). Scherr et al. (2013) reported that a target RPE scale score of $11-13$ is appropriate for less-trained individuals, increas- ing to $13-15$ when more intense training is necessary. In the present study, the RPE scale scores ranged from 9 to 14 in all exercise conditions. Although the curl-up with maximum expiration required greater effort than that with slow expiration, the difficulty of the two types of exercise was similar (i.e., moderate). In classifying muscle activity, a previous study considered $0 \%-20 \%$ maximum voluntary contraction to correspond to low muscle activity, while $21 \%-40 \%$ was moderate, $41 \%-60 \%$ was high, and greater than $60 \%$ was very high. In this study, regardless of the hip position, the curl-up with maximum expiration elicited high muscle activity, and thus can be recommended for abdominal strength training.

This study had several limitations. First, there was a lack of EMG data on the other hip muscles, such as the gluteus maximus, which may also be influenced by the hip position during exercise. Second, our results cannot be generalized to other populations because of the limited number of subjects included in the study. Finally, kinematic changes in the pelvis could not be measured. Further investigations addressing these limitations are warranted.

In summary, both the hip position and breathing pattern affect abdominal muscle activities during the curl-up exercise, especially of the oblique muscles. Among the breathing patterns investigated herein, the curl-up with maximum expiration produced greater activation of the oblique muscles compared to slow expiration and maximum inspiration. The curl-up with $90^{\circ}$ hip flexion was effective in activating the $\mathrm{IO}$ and $\mathrm{EO}$, regardless of the breathing pattern. 


\section{CONFLICT OF INTEREST}

No potential conflict of interest relevant to this article was reported.

\section{ACKNOWLEDGMENTS}

This work was supported by Kaya University.

\section{REFERENCES}

Abe T, Kusuhara N, Yoshimura N, Tomita T, Easton PA. Differential respiratory activity of four abdominal muscles in humans. J Appl Physiol (1985) 1996;80:1379-1389.

Andersson EA, Nilsson J, Ma Z, Thorstensson A. Abdominal and hip flexor muscle activation during various training exercises. Eur J Appl Physiol Occup Physiol 1997;75:115-123.

Burden AM, Redmond CG. Abdominal and hip flexor muscle activity during 2 minutes of sit-ups and curl-ups. J Strength Cond Res 2013; 27:2119-2128.

Cho KH, Beom JW, Lee TS, Lim JH, Lee TH, Yuk JH. Trunk muscles strength as a risk factor for nonspecific low back pain: a pilot study. Ann Rehabil Med 2014;38:234-240.

Comfort P, Pearson SJ, Mather D. An electromyographical comparison of trunk muscle activity during isometric trunk and dynamic strengthening exercises. J Strength Cond Res 2011;25:149-154.

Cram JR, Kasman GS, Holtz J. Introduction to surface electromyography. Maryland: Aspen; 1998.

Czaprowski D, Afeltowicz A, Gębicka A, Pawłowska P, Kędra A, Barrios C, Hadała M. Abdominal muscle EMG-activity during bridge exercises on stable and unstable surfaces. Phys Ther Sport 2014;15:162-168.

Davidson KL, Hubley-Kozey CL. Trunk muscle responses to demands of an exercise progression to improve dynamic spinal stability. Arch Phys Med Rehabil 2005;86:216-223.

Gidaris D, Hatzitaki V, Mandroukas K. Spinal flexibility affects range of trunk flexion during performance of a maximum voluntary trunk curl-up. J Strength Cond Res 2009;23:170-176.
Hartvigsen L, Kongsted A, Hestbaek L. Clinical examination findings as prognostic factors in low back pain: a systematic review of the literature. Chiropr Man Therap 2015;23:13.

Ishak NA, Zahari Z, Justine M. Effectiveness of strengthening exercises for the elderly with low back pain to improve symptoms and functions: a systematic review. Scientifica (Cairo) 2016;2016:3230427.

Ishida $\mathrm{H}$, Watanabe $\mathrm{S}$. Maximum expiration activates the abdominal muscles during side bridge exercise. J Back Musculoskelet Rehabil 2015;28:81-84.

Kim HD, Jeon DM, Bae HW, Kim JG, Han N, Eom MJ. Changes in activation of abdominal muscles at selected angles during trunk exercise by using ultrasonography. Ann Rehabil Med 2015;39:950-956.

Lee DK, Moon DC, Hong KH. Effect of neck flexion restriction on sternocleidomastoid and abdominal muscle activity during curl-up exercises. J Phys Ther Sci 2016;28:90-92.

Martuscello JM, Nuzzo JL, Ashley CD, Campbell BI, Orriola JJ, Mayer JM. Systematic review of core muscle activity during physical fitness exercises. J Strength Cond Res 2013;27:1684-1698.

Muscolino JE. Know the body: muscle, bone, and palpation essentials. Maryland Heights (MO): Elsevier Mosby; 2012.

Parfrey KC, Docherty D, Workman RC, Behm DG. The effects of different sit- and curl-up positions on activation of abdominal and hip flexor musculature. Appl Physiol Nutr Metab 2008;33:888-895.

Rutkowska-Kucharska A, Szpala A. Electromyographic muscle activity in curl-up exercises with different positions of upper and lower extremities. J Strength Cond Res 2010;24:3133-3139.

Rutkowska-Kucharska A, Szpala A. The use of electromyography and magnetic resonance imaging to evaluate a core strengthening exercise programme. J Back Musculoskelet Rehabil 2018;31:355-362.

Scherr J, Wolfarth B, Christle JW, Pressler A, Wagenpfeil S, Halle M. Associations between Borg's rating of perceived exertion and physiological measures of exercise intensity. Eur J Appl Physiol 2013;113:147155.

Yoon TL, Kim KS, Cynn HS. Slow expiration reduces sternocleidomastoid activity and increases transversus abdominis and internal oblique muscle activity during abdominal curl-up. J Electromyogr Kinesiol 2014;24:228-232. 